\title{
Mobile Computing: Review
}

\author{
Dr. Krishan Pal, Sachin Raj Saxena* \\ Computer science \& Engineering, Future College Bareilly
}

*Corresponding Author: Sachin Raj Saxena, Computer science \& Engineering, Future College Bareilly

\begin{abstract}
In this paper the use of technology is pervasive. We are discussing on mobile computing its types and current trends of mobile computing, technical era Mobile computing is latest as a technology that allows transmission of data, voice and video and improved understanding of human mobility patterns would yield insights into a variety of important societal and urban planning issues. The integration of mobile computing and sensing with cloud computing enables new applications that process big data collection and identify important places people visit, infer relative traffic volumes on city roads. This paper involves with the main concept of trends, technology, mobile communication and mobile hardware.
\end{abstract}

Keywords: Band width1, Devices2, Data dissemination3 and principles of mobile computing Mobile; Mobile computing; Wireless communication;Portable devices.

\section{INTRODUCTION}

Mobile Computing is a technology that allows transmittance of data, voice and video via a computer or any other on the air enabled contrivance without having to be connected to a fixed physical link. The main concept involves -

i)Mobile communication

ii)Mobile hardware

iii)Mobile software

\subsection{Mobile Communication}

The mobile communication pertains to the base put in place to ensure that seamless and reliable communication goes on. the aforesaid would embrace contrivance such as protocols, services, bandwidth, and portals imperative to expedite and assistance the aforesaid services. The data makeup is also defined at this parascene. This ensures that there is no percussion with other existing systems which offer the same service. Since the media is unguided/unbounded, the over laying substructure is fundamentally radio wave- oriented. That is, the signals are led over the air to unintended devices that are adequate to of consignee and consignment and similar kinds of signals.

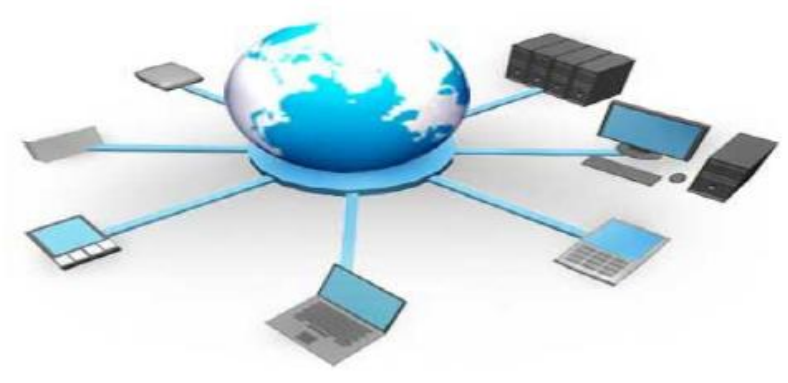

\subsection{Mobile Hardware}

Mobile hardware embraces mobile apparatuses or device facets that receive or access the service of mobility. They would range from portable laptops, smartphones, tablet Pc's, Personal Digital Assistants.

These devices will have a sensory receptor medium that is capable of perception, spotting and receiving signals. These devices are setup to operate in full- duplex, whereby they are equal to sending and receiving signals at the same time. They don't have to wait until one device has finished disclose for the other gimmick to enlightened communications. 


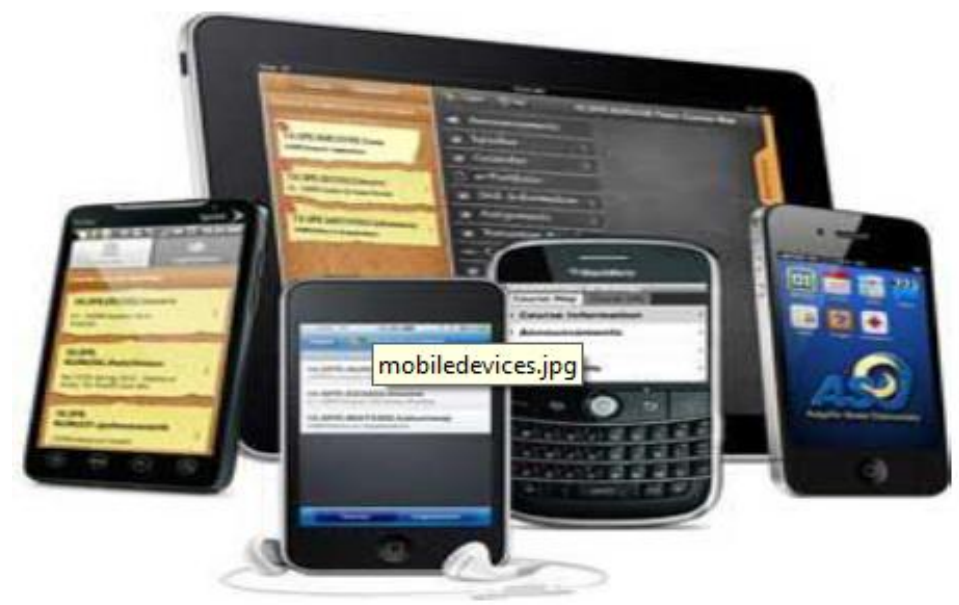

\subsection{Mobile Software}

Mobile software is the real program that runs on the mobile hardware. It treats with the bargains and provisions of mobile diligence. This is the locomotive of the mobile device. In other terms, it is the operating system of the gadget. It's the essential component part that operates the mobile device.
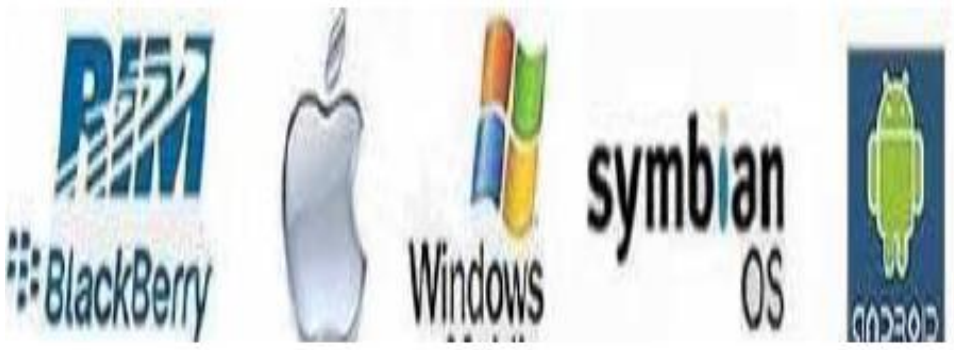

Since transferal is the majority ingredient, this kind of computing ensures that users are not tied or fast to a single fix location, but are easy to dab from anywhere. It includes all care of wireless communications.

\section{Mobile Computing - Classification}

Mobile computing is not only finite to mobile phones, but there are many devices vacant in the market that are built on a floor to support mobile computing. They are usually private in the following class

\subsection{Personal Digital Assistant (PDA)}

The main plan of this device is to act as an electronic promoter or day creator that is portable, easy to use and able of allocation information with your computer systems.

PDA is an delay of the PC, not a stand-in. These systems are able of sharing information with a computer system through a system or service known as harmony. Both devices will access each other to check for changes or renew in the lone devices. The use of infrared and Bluetooth connections enables these devices to always be synchronized.

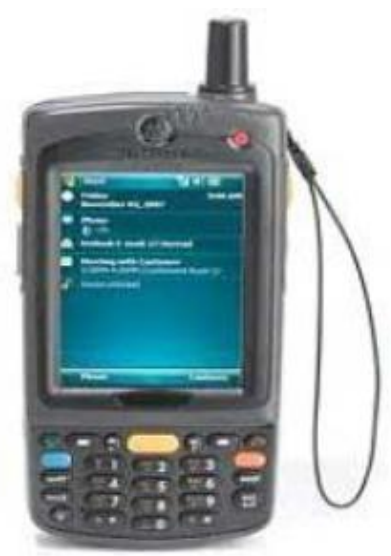




\subsection{Smartphones}

This class of phone bind the features of a PDA with that of a mobile phone or camera phone. It has a choice edge over other class of mobile phones.

These phones add high-resolution touch screens, web browsers that can access and well display standard web pages rather than just mobile-optimized sites, and high-speed data path via Wi-Fi and high speed cellular broadband. The most regular mobile Operating Systems (OS) used by modern smartphones add Google's Android, Apple's iOS, Nokia's Symbian, RIM's BlackBerry OS, Samsung's Bada, Microsoft's Windows Phone, and embedded Linux distributions such as Maemo and MeeGo. These operating systems can be connected on various phone models, and typically each agent can receive various OS software updates over its period.
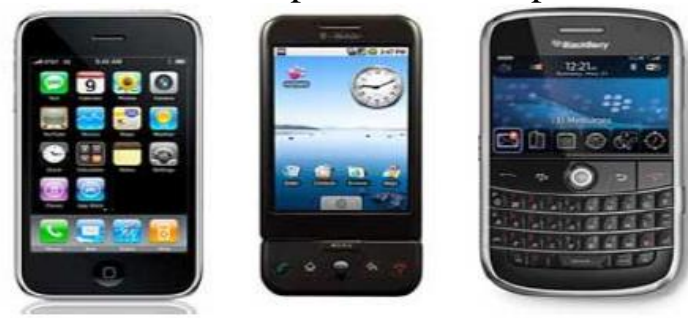

\subsection{Tablet PC and Ipads}

This mobile appliance is better than a mobile phone or a PDA and arrange into a touch screen and is operated using touch seeing motions on the screen. They are often calm by a pen or by the touch of a finger. They are normally in record form and are light in weight. Examples would add ipads, Galaxy Tabs, Blackberry Playbooks etc

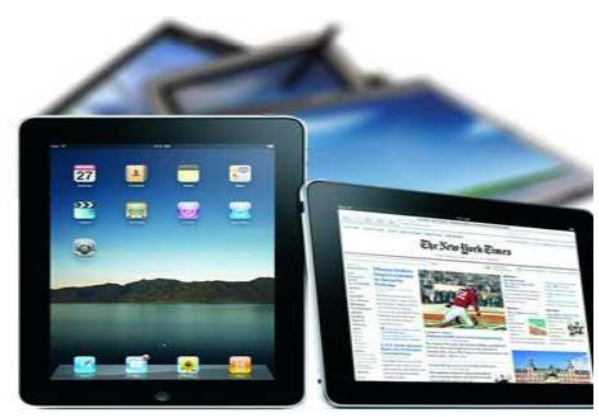

They bid the same use as compact computers. They collar mobile computing in a deep choice way and have gross alter force. Users can cut and redo log files, door high speed internet, stream video and audio data, receive and send e-mails, attend/give lectures and show mid its so many other part.

\section{Mobile Computing - Major Advantages}

Mobile computing has replace the all landscape of our day-to-day life. latter are the big aid of Mobile Computing -

1.Location Flexibility-This has fit end user to effort taken away anywhere as long as there is a ally fixed. A buyer can work without being in a fixed spot. Their mobility secure that they are good to carry out big tasks at the same time and meet their told jobs.

2.Saves Time-The pace vanish or wasted while carry from various site or to the work and back, has been rip. One can now path all the large form and files over a protected canal or entry and work as if they were on their computer. It has pad telecommuting in many companies. It has also shrunk useless earn costs.

3.Enhanced Productivity-End user can work nicely and finally from that location they find hale. This in turn pad their production level.

4.Ease of Research-Probe has been made simple, since users earlier were set to go to the field and search for poop and hay them back into the system. It has also made it simple for field officers and researchers to amass and feed data from wherever they are without making useless trips to and from the office to the field. 


\section{Mobile COMPUTING - SECURITY ISSUES}

Mobile computing has its fair share of security concerns as any other technology. Due to its pastoral nature, it's not easy to guide the proper formula. Users clout have different goal on how to apply this claim. Odd and wrong rule such as break, technical tailing, pirating, online con and malicious murder are some but few of the trouble sport by mobile computing.

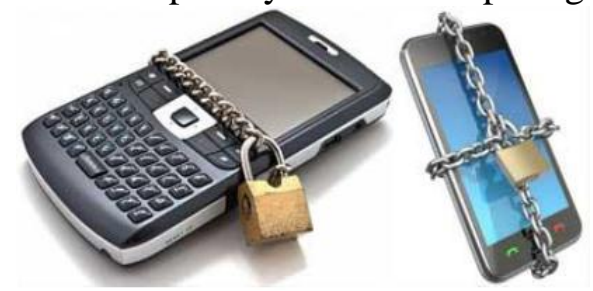

No company need to lay open their code to hackers and other intruders, who will in turn sell the valuable lore to their rival. It's also large to take the basic care to pan these risk from taking place. Some of those part add -

- Rent qualified personnel.

- Lay security hardware and software

- Rear the users on proper mobile computing ethics

- Auditing and developing sound, effective policies to govern mobile computing

- Lash proper access rights and permissions

In the cut of such bulk, it's cinch for feat and other far threats to tinge and cause sure harm. These may be in terms of credit or pocket penalties. In such cases, it's very easy to be wrong in different scam practices.If these board aren't well worked on, it clout be an alley for uniform threat. Various threats still exist in enable this humane of technology.

\section{Mobile Computing - Current Trends}

Mobile computers starting from 3G technologies which is the hottest mobile technology on hand in the market.

\subsubsection{G}

$3 \mathrm{G}$ or third generation mobile is a generation of test for mobile phones and mobile telecommunication services event the International Mobile Telecommunications-2000 (IMT- 2000) specifications by the International Telecommunication Union. Action services add wide-area wireless voice telephone, mobile Internet path, video calls and mobile TV, all in a mobile environment.

\subsection{Global Positioning System (GPS)}

The Global locate System (GPS) is a space-based moon navigation system that add location and time lore in all weather, all over on near the Earth, where there is an free line of view to four or more GPS planetoid. The GPS program add demanding means to force, civil and commercial buyer around the world. GPS is the backbone for improve the global air traffic system, resist, and location services.

\subsection{Long Term Evolution (LTE)}

LTE is a standard for wireless contact of high-speed data for mobile phones and data depot. It is based on the GSM/EDGE and UMTS/HSPA network technologies, waxing the capacity and speed using new tone techniques. It is related with the use of fourth Generation (4G) technology.

\subsubsection{G}

The 5G mobile cellular communications system add a far higher level of act than the past generations of mobile communications systems. The new 5G technology is not just the next version of mobile communications, grow from $1 \mathrm{G}$ to $2 \mathrm{G}, 3 \mathrm{G}, 4 \mathrm{G}$ and now $5 \mathrm{G}$.Rather $5 \mathrm{G}$ technology is very various. Past systems had evolved driven more by what could be done with the new technology. The new $5 \mathrm{G}$ technology has been driven by set uses applications.5G has been driven by the need to add ubiquitous connectivity for functions as diverse as auditive communications, remote control with hematic style 
answer, huge video downloads, as well as the very low data rate functions like remote sensors and what is being termed the IoT, Internet of Things.

\subsection{WiMAX}

WiMAX (Worldwide Interoperability for Microwave Access) is a wireless communications test form to add 30 to

40megabit-per-second data rates, with the new update adding up to $1 \mathrm{Gbit} / \mathrm{s}$ for fixed locus. It is a part of a fourth generation or 4G wireless-communication technology. WiMAX far beat the 30metre wireless range of a conventional Wi-Fi Local Area Network (LAN), offering a metropolitan area network with a signal radius of about $50 \mathrm{~km}$. WiMAX offers data move that can be choice to current cable-modem and DSL connections, however, the bandwidth must be joint among different users and thus yields lower speed in rule.

Consumers are most likely to encounter WiMAX products and services in "last mile" applications. WiMAX allows ISPs and carriers to provide Internet connectivity to homes and offices without requiring the use of physical wiring (copper, cable, etc.) leading to the customer's premises.

WiMAX is often compared to Wi-Fi; both have wireless Internet connectivity at their core, and the technologies are complementary. Primary similarities and differences include:

- WiMAX's range is measured in kilometers, while Wi-Fi is measured in meters and local in nature. WiMAX's reliability and range make it suitable for the provision of Internet access covering large metropolitan areas.

- Wi-Fi uses an unlicensed spectrum, while WiMAX's spectrum could be licensed or unlicensed.

- Wi-Fi is more popular in end-user devices such as laptops, desktops and smart phones. Accordingly, WiMAX service providers usually provide the customer with a WiMAX subscriber unit. This unit connects to the provider's network and offers Wi-Fi accessibility and convenience to the customer within the Wi-Fi range.

\section{CONCLUSION}

Mobile computing is human-computer communication in which a computer is normal to be carried at the time normal usage, which grant for sending of data, voice and video. Mobile computing hold mobile communication, mobile hardware, and mobile software.Today's computing has fast grown from being cramp to a single location. With mobile computing, people can work from the cheer of any location they wish to as long as the connection and the security care are well split. In the same light, the being of high speed ally has also puff the use of mobile computing.Being an ever keep and flow technology, mobile computing will reach to be a core avail in computing, and Information and means technology.An overview of Mobile Computing and then it will take you over how it grow and where is the technology going to in future onward with the classes and security issues confusing. Video and audio recordings can now be run on-the-go using mobile computing. It's easy to path a wide change of movies, academic and chatty material. With the advance and show of high speed data ally at lavish cost, one is able to get all the entertainment they want as they browse the internet for flood data. One is able to watch news, movies, and film among other entertainment offers over the internet. This was not set up before mobile computing loom on the computing nature.

\section{REFERENCES}

[1] Journals publication paper, 2014-2016 Final Year Projects | Mobile Computing.

[2] T Grew hill in 2009 paper publication, its technical events are gathered.

[3] Journal >>IEEE Transactions on mobile computing. Locate articles and query publisher details, 2015 Journal impact 3.74 .

[4] http://www.researchgate.net/../1536-1233_IEEE_Transactions_on_MobileComputing.

[5] C. Hewitt, "Orgs for scalable, robust, privacy-friendly client cloud

[6] Computing," Internet Computing, IEEE, vol. 12, no. 5, pp. 96-99, 2008.

[7] R. Buyya, C. Yeo, and S. Venugopal, "Market-oriented cloud computing:Vision, hype, and reality for delivering it services as computing utilities,"in High Performance Computing and Communications, 2008. HPCC'08. 
[8] Agrawal, D.,\& Zeng, A. (2006). Introduction to Wireless and Mobile Systems, 2nd edition, Hampshire, UK.Thomson Learning.

[9] Helal, A. (2002). Anytime, Anywhere Computing : Mobile Computing Concepts and Technology. SECS 522. Kluwer Academic. EBook. Web.

[10] Kwok, Y., \& Lau, V. (2007). Wireless Internet and Mobile Computing: Interoperability and Performance. New Jersey. Wiley-IEEE Press.

[11] Mallick, M. (2003). Mobile and Wireless Design Essentials. New Jersey. Wiley Publishing.

[12] Reza ,B. (2005). Mobile Computing Principles: Designing and Developing Mobile Applications with UML and XML. Cambridge, UK. Cambridge University Press

[13] Agrawal, D.,\& Zeng, A. (2006). Introduction to Wireless and Mobile Systems, 2nd edition, Hampshire, UK.Thomson Learning.

[14] Helal, A. (2002). Anytime, Anywhere Computing : Mobile Computing Concepts and Technology. SECS 522. Kluwer Academic. EBook. Web.

[15] Kwok, Y., \& Lau, V. (2007). Wireless Internet and Mobile Computing: Interoperability and Performance. New Jersey. Wiley-IEEE Press.

[16] Mallick, M. (2003). Mobile and Wireless Design Essentials. New Jersey. Wiley Publishing.

[17] ]Reza ,B. (2005). Mobile Computing Principles: Designing and Developing Mobile Applications with UML and XML. Cambridge, UK. Cambridge University Press.

Citation: Sachin Raj Saxena \& Dr. Krishan Pal, (2019). "Mobile Computing: Review”. International Journal of Research Studies in Computer Science and Engineering (IJRSCSE), 6(4), pp.1-6. http://dx.doi.org/10.20431/2349-4859.0604001

Copyright: () 2019 Authors, this is an open-access article distributed under the terms of the Creative Commons Attribution License, which permits unrestricted use, distribution, and reproduction in any medium, provided the original author and source are credited. 